\title{
Conservative management of bilateral traumatic testicular dislocation in a 10-year-old boy
}

\author{
Yuko Shirono $^{1}$, Shunsuke Yamaguchi ${ }^{1}$, Eisuke Takahashi ${ }^{1}$, and Masahiro Terunuma ${ }^{1}$ \\ ${ }^{l}$ Department of Urology, Nagaoka Chuo General Hospital, Japan
}

\begin{abstract}
A 10-year-old boy fell from a one-meter-high Jacuzzi ladder in a hot spring facility, landing in a straddle position, and injured his perineum. He visited the emergency room of our hospital immediately after the injury. Magnetic resonance imaging (MRI) revealed a tear of the corpus spongiosum urethra, and compression due to a hematoma. With the hematoma spreading to the scrotum, the testes became inverted and dislocated to the inguinal region on both sides. Without surgery or interventions, the testes descended into the scrotum on the third day after the injury before fibrillation and scarring began. Testicular dislocation by injury is rare and encountered exclusively in children. It is generally treated with surgery to retain testicular function. We selected conservative management, as our patient had a closed injury without testicular torsion, and the testicular dislocation was associated with compression by hematoma, which could possibly recover with regression of the hematoma.
\end{abstract}

Key words: testicular dislocation, inguinal dislocation, straddle injury, conservative management, pediatric patient

(J Rural Med 2018; 13(1): 82-85)

\section{Introduction}

Traumatic testicular dislocation (TTD) is an injury where the testes are displaced out of the scrotum by an external force, and TTD is relatively rare among genito-urinary tract traumas. In the case of early diagnosis, a few reports revealed that manual reduction succeeded, but in many cases,

Received: October 26, 2017

Accepted: January 18, 2018

Correspondence: Yuko Shirono, Department of Urology, Nagaoka Chuo General Hospital, Kawasaki-machi 2041, Nagaoka, Niigata 9408653, Japan

E-mail: yuko-shirono@med.niigata-u.ac.jp

This is an open-access article distributed under the terms of the Creative Commons Attribution Non-Commercial No Derivatives (by-nc-nd) License $<\mathrm{http}$ //creativecommons.org/licenses/by-nc-nd/4.0/>. an open reduction may be required ${ }^{1-3)}$. Our report addresses the use of conservative management in a case of inguinal testicular dislocation in a pediatric patient whose testes descended into the scrotum.

\section{Case Report}

A 10-year-old boy complaining of perineal trauma was admitted. He had no remarkable medical history and had never been diagnosed with cryptorchidism or migratory testis on medical examination. He fell from a one-meter-high Jacuzzi ladder and injured his perineum in a straddle position. He visited the emergency room of our hospital immediately after the injury.

A subcutaneous hematoma was found in the perineum; the scrotum was dark red and enlarged (Figure 1). He had a micturition feeling, but could not urinate. No bleeding from the external urethral meatus was observed. Ultrasonography (US) showed the absence of testes in the scrotum. Magnetic resonance imaging (MRI) revealed a fissure and hematoma in the corpus spongiosum urethra at the level of the bulbous urethra, with the hematoma filling the scrotum, and inverted testes on both sides of the inguinal regions (Figure 2).

Although urethrography showed severe stenosis in the bulbous urethra, contrast medium flowed in a filamentous form to the bladder, and no obvious urethral damage was observed (Figure 3 ). When a 12 French urinary catheter was inserted, $160 \mathrm{ml}$ of yellow urine flowed out.

We attempted manual reduction, but it was difficult, due to pain and the hematoma filling the scrotum. After informed consent was obtained, a few days of conservative management without surgery as the method of treatment.

Three days after the injury, US revealed that the bilateral testes were in the upper part of the scrotum, and blood flow was found in the testes on color-flow Doppler US. Four days after the injury, MRI revealed a decreased hematoma in the 
a

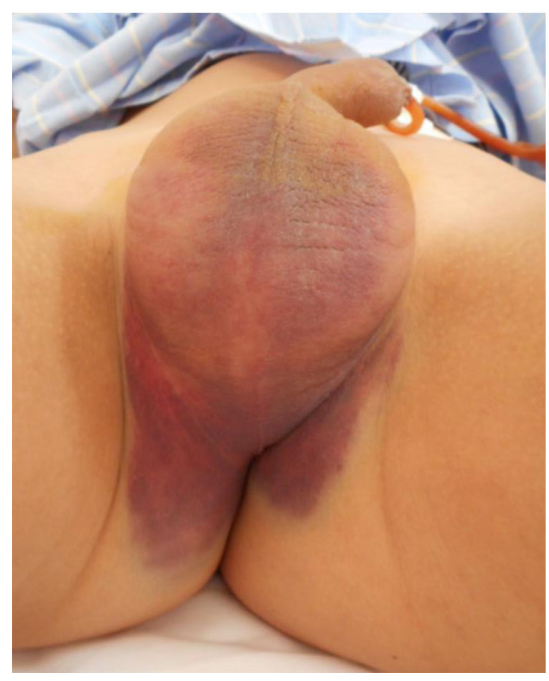

b

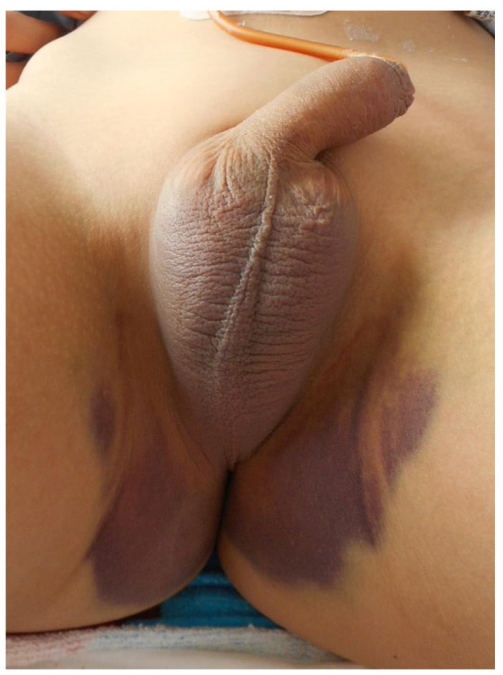

C

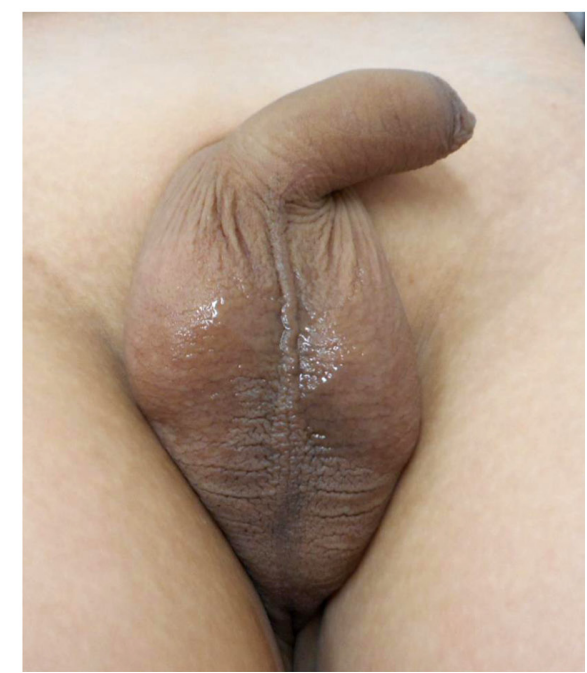

Figure 1 Physical examination findings. a. One day after the injury b. Seven days after the injury c. Six months after the injury.

a

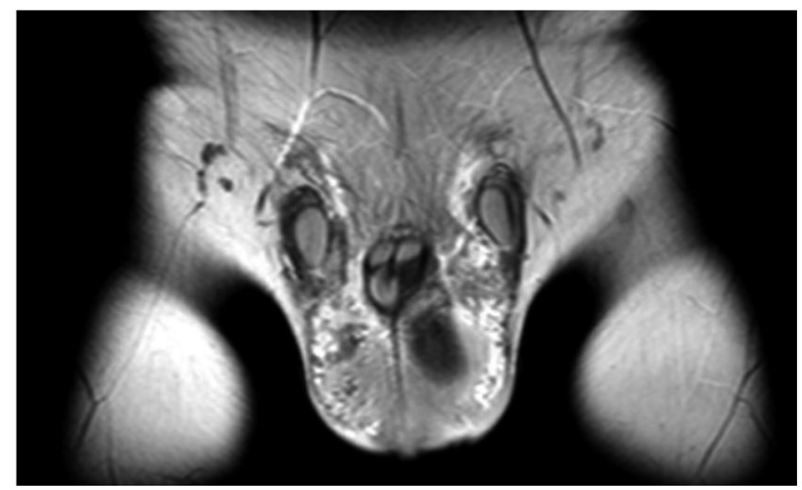

b

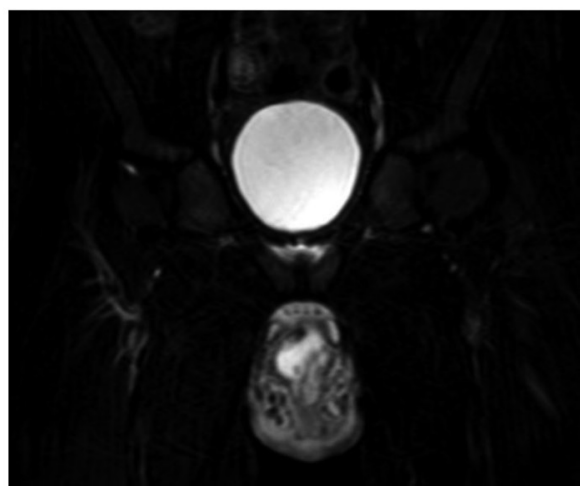

Figure 2 Magnetic resonance imaging findings immediately after the injury. a. A hematoma spread throughout the scrotum, and the testes were inverted on both sides of the inguinal regions. b. A fissure and hematoma in the corpus spongiosum urethra were found at the level of the bulbous urethra.

scrotum, bilateral testes in the upper part of the scrotum, and no hematoma in the corpus spongiosum urethra (Figure 4). Grossly with physical examinations, the hematoma at the perineum expanded to a butterfly shape and peaked seven days after the injury, but later turned yellow and disappeared. The swollen scrotum contracted over time and returned to a normal size at the time of discharge (Figures $1 \mathrm{a}-\mathrm{c})$. Twelve days after the injury, the urinary catheter was removed and the patient did not complain of dysuria. He was discharged from the hospital on the 18th day after the injury.

\section{Discussion}

TTD is a state in which external force is exerted directly or indirectly and the testis is displaced outside the scrotum. In most cases, the Alyea ${ }^{4}$ classification of trauma is widely used and categorizes TTD as a superficial, internal, or complex dislocation (Figure 5). Superficial dislocation is the displacement of the testis somewhere along a circle within the radius of the spermatic cord and centered on the external inguinal ring. This distinction can be further divided into the inguinal, pubic, perineal, penile, and crural types.

Internal dislocation involves the testes moving backward from the scrotum to the inguinal ring and the inguinal 


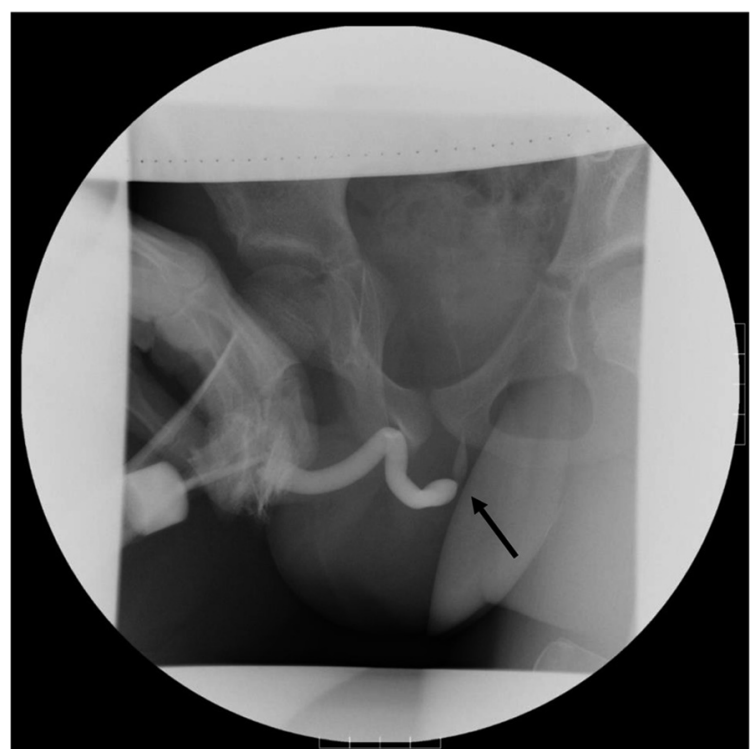

Figure 3 Urethrography showed strong stenosis in the bulbous urethra.

canal, the intra-abdominal, and the femoral canal. Complex dislocation is translocation of the testes from the open wound of the scrotum to the outside of the scrotum. The most common site of dislocation is the superficial inguinal pouch (almost $50 \%$ of all cases). Other less common sites of TTD include: pubic (18\%), penile (8\%), canalicular (8\%), truly abdominal (6\%), perineal (4\%), acetabular (4\%), and crural $(2 \%)^{5}$. More than half of the causes of onset are traffic injuries, mostly caused by motorcycle accidents. Injuries caused by soccer, skiing, bowel movements, and exercise abuse have also been reported ${ }^{1)}$.

Congenital vulnerability of testicular fixation to the inferior scrotum has been reported ${ }^{6}$. In superficial dislocation, external force and spasm of the testicular cremaster muscle are applied, causing testicular dislocation. Internal dislocation is believed to occur when the testes are small and the inguinal ring and canal are open ${ }^{7}$. According to Igarashi et al.'s report, abnormal adherence of the gubernaculum testis was observed in two out of four cases in which surgery was performed ${ }^{11}$. According to Inoue et al.'s report, there is an excessive length of the gubernaculum testis, suggesting the possibility of migratory testis ${ }^{6}$.

When testicular dislocation is suspected, MRI and US are useful for early diagnosis ${ }^{2}$. In some cases, torsion of the spermatic cord is merged with dislocation, and evaluation of testicular blood flow is necessary.

Edema occurs in the pathway after testicular dislocation, and the scarring substance progresses around the testis from the fourth day of the injury, so manual reduction becomes difficult over time ${ }^{8)}$. When the testes are necrosed because of torsion of the spermatic cord, or little improvement is achieved with long-term observations after trauma, orchiectomy should be considered.

It has been suggested that patients with a closed injury whose testicular dislocation was possibly caused by compression from a hematoma, without testicular torsion during the early posttraumatic period (within four days), can be managed without surgery ${ }^{1,8)}$. Surgery may be recommended for those with a complicated injury, testicular torsion, little improvement with long-term observations, or difficulty in manual reposition despite the absence of hematoma/edema.

Besides testicular trauma, caution is required for com- a

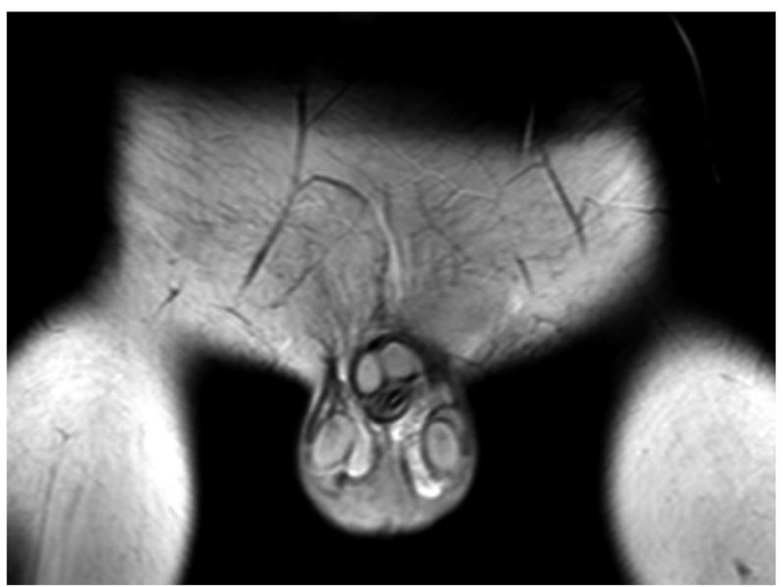

b

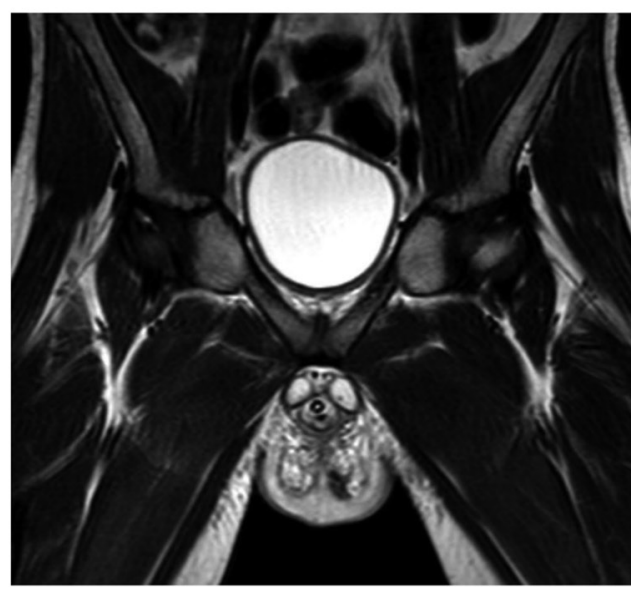

Figure 4 MRI findings four days after injury. a. The hematoma decreased in the scrotum; bilateral testes were found in the upper part of the scrotum. $b$. The hematoma in the corpus spongiosum urethra disappeared. 


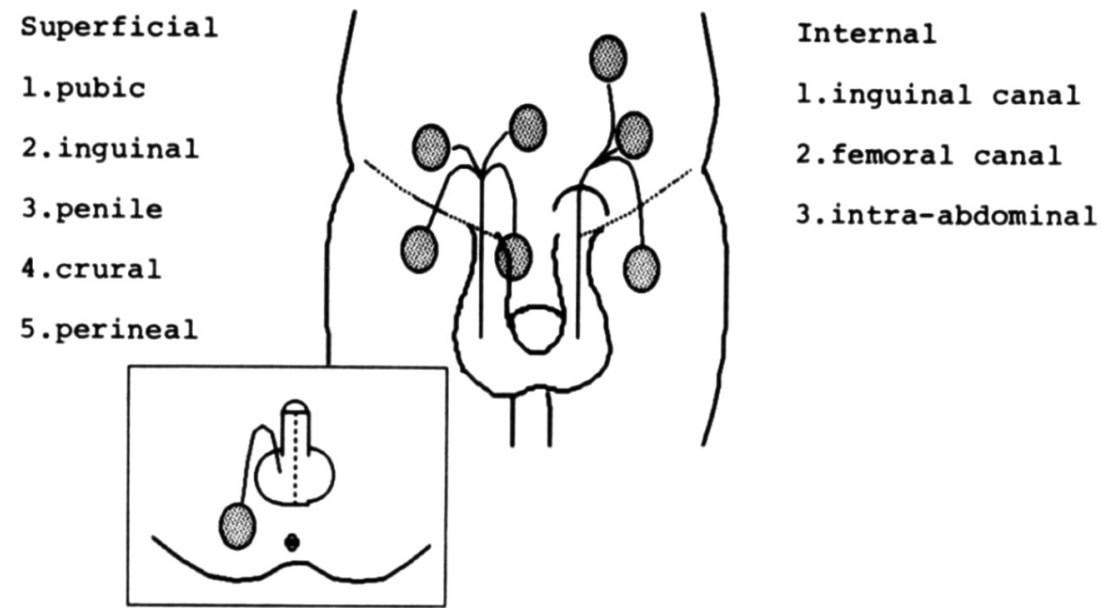

Figure 5 The Alyea classification

plications of urethral injury, traumatic priapism, and bladder rupture in the treatment of trauma. Next, a treatment approach will be considered for functional preservation. Because young people often have scrotum and testicular trauma, consideration of fertility is necessary.

Some cases such as atrophy of seminiferous tubules were recognized in cases wherein histopathological examination was performed ${ }^{1,3)}$. However, this includes cases that have a long period of time from injury to surgery, and histopathological examination is not performed when open reduction is performed early after the injury. The effect of TTD on testicular function is unknown.

\section{Conclusion}

Testicular dislocation is a rare event in testicular trauma, often requiring surgical reduction. But in the case of early diagnosis, it can be managed without surgery, when certain conditions are satisfied.

The abstract of this paper was presented at the 66th Annual Meeting of the Japanese Association of Rural Medicine.

There are no conflicts of interest associated with this publication, and there has been no significant financial support for this work that could have influenced its outcome.

\section{References}

1. Atsushi I, Toshio K, Kazuo W, et al. Traumatic dislocation of the testis: report of five cases. Hinyokika Kiyo (Acta Urol Jpn) 2003; 49: 603-605 (in Japanese, Abstract in English).

2. Zavras N, Siatelis A, Misiakos E, et al. Testicular dislocation after scrotal trauma: a case report and brief literature review. Urol Case Rep 2014; 2: 101-104. [Medline] [CrossRef]

3. Masahiro I, Akitoshi T, Teiichiro U. Traumatic intraperitoneal testicular dislocation: a case report. Hinyoki Geka 2015; 28: 985-988 (in Japanese, Abstract in English).

4. Alyea EP. Dislocation of testis. Surg Gynecol Obstet 1929; 49: 600-616.

5. Perera E, Bhatt S, Dogra VS. Traumatic ectopic dislocation of testis. J Clin Imaging Sci 2011; 1: 17. [Medline]

6. Katsumi I, Tsuneo M, Kazuo W, et al. A case of testicular luxation. Hinyoki Geka 1998; 11: 269-271 (in Japanese, Abstract in English).

7. OConnell R, Hargan B, Murphy DM, et al. Traumatic dislocation of the testis. Ir Med J 1984; 77: 107-108. [Medline]

8. Herbst RH, Polkey HJ. Luxatio testis traumatica. Am J Surg 1936; 34: 18-40. [CrossRef] 